

\title{
Symposium grafica 2020 Graphics Symposium 2020
}

Daniel Tena-Parera. Universitat Autònoma de Barcelonal, daniel.tena@uab.cat

\begin{abstract}
Resumen
En esta editorial relatamos lo acontecido en el VI Symposium grafica que, debido a la presencia del COVID-19, se celebró a través de la plataforma digital Teams. Tres conferencias y más de 14 intervenciones entre ponencias y exposiciones permitieron experimentar con los instrumentos digitales y demostraron que lo importante es el interés en participar. Participación que no hubiera sido posible de no ser por un reducido grupo de personas que persiguen poner en común su contribución. Se cerró el Symposium grafica del 2020 y se anunció ya el $7^{\circ}$ Symposium grafica.

Palabras clave

Publicidad; diseño gráfico; Symposium grafica
\end{abstract}

\begin{abstract}
In this editorial we report what happened in the VI Graphic Symposium that under the presence of COVID-19 was held through the digital platform Teams. Three conferences and more than 14 interventions between presentations and exhibitions, allowed to experiment with digital instruments and showed that the important thing is the interest in participating. Participation that would not have been possible had it not been for a small group of people who seek to pool their contribution. The 2020 Graphic Symposium was closed and the 7th Graphic Symposium was announced.
\end{abstract}

Keywords

Advertising; graphic design; grafica Symposium

\section{EDITORIAL pp. 05-09}


El Symposium grafica 2020 fue una realidad a pesar de la pandemia covid-19. EI VI Symposium grafica, SEXTO ENCUENTRO ACADÉMICO DE INVESTIGACIÓN EN DISEÑO GRÁFICO se celebró los días 2, 3 y 4 de septiembre de 2020. El anfitrión de esta edición fue, con motivo de su $15^{\circ}$ Aniversario, el Col-legi Oficial de Disseny Gràfic de Catalunya, "la "única corporación de derecho público que vela por la defensa de los intereses del diseño de la comunicación visual, sus profesionales y usuarios".

Como siempre, el symposium se dirige al mundo universitario y profesional. Por ello, profesores, estudiantes, investigadores y profesionales del diseño gráfico y creatividad publicitaria participan anualmente en el evento.

A diferencia de las 5 Ediciones anteriores del Symposium grafica, en las que el Symposium fue presencial, en esta $6^{a}$ Edición, dada la situación generada por la pandemia del Covid-19, fue en formato online.

Por tanto, el $6^{\circ}$ Symposium grafica estuvo formado por las conferencias, actos y defensa de comunicaciones online que, durante los días 2, 3 y 4 de setiembre de 2020, nos permitieron dialogar sobre el Diseño gráfico, la Comunicación, la Creatividad y la Publicidad.

Aunque se realizó en modo online, el concepto de symposium fue el mismo. Los ponentes dieron sus charlas y los asistentes pudieron interactuar con ellos. La única diferencia fue que se realizó a través de la plataforma virtual "Teams".

\section{EL COMPROMISO}

Si bien es cierto que lo material mueve el espíritu de las personas, cuando hablamos de ideales, de objetivos comunes, de pensamientos sobre las cosas, hay una fuerza adicional que impulsa al ser humano a superarse.

Creo que el compromiso del diseño gráfico y de la creatividad, de las personas que se dedican al diseño gráfico y a la creatividad se les genera un impulso para con lo suyo y para con lo de los demás que produce dinámicas insospechadas.

Esto fue lo que sucedió en el VI Symposium grafica 2020. Ante las dificultades evidentes producidas por una pandemia de la que todavía no se ve, en el momento de escribir estas líneas, su final; una fuerza manifiesta producida por los participantes, impulso el debate para convertirlo en un auténtico compromiso. El del diseño y el de las personas vinculadas a él, que se debe seguir adelante. Que el diseño y la creatividad están al servicio no del diseño ni de la creatividad ni de quien los realiza, sino de las personas. $Y$ ello nos obliga, a todos, a trabajar por la mejora del ámbito del diseño gráfico y la creatividad.

Y fue posible gracias a la oculta complicidad de personas que a través de la plataforma digital "Teams", permitieron poner en contacto a los más de cuarenta participantes en el symposium y que en distintos momentos pudieron disfrutar de las perspectivas de sus colegas.

\section{CONFERENCIAS VIRTUOSAS}

Fue la primera vez que en el symposium se programaron tres conferencias que recayeron en académicos virtuosos de su trabajo.

La conferencia inaugural la realizó el profesor y doctor de la "University of Puerto Rico", José Ayala. La conferencia central corrió a cargo del Doctor José Javier González Solas, profesor jubilado de la "Universidad Complutense de Madrid". Finalmente, la conferencia de clausura la dictó Teresa Camacho profesora y en la Universidad Anáhuac Querétaro y que, desde 1994, trabaja como free lance para empresas e instituciones.

Perfiles innovadores del Diseño Creativo. - Con este sugerente título, José Ayala expuso la perspectiva sobre el diseño donde la coincidencia de la innovación es disruptiva con los tradicionales perfiles profesionales (Fig. 1). La creatividad, el diseño, los creativos, los diseñadores y la realidad profesional donde la innovación es sinónimo de avance ponen en cuestión la consideración tradicional y antropológica del diseño. Visión en la que el diseñador, el creativo son generadores de nuevas formas para el funcionamiento de la sociedad. Los perfiles hegemónicos no son los que diseñan y crean. Los perfiles hegemónicos son los que dominan el medio digital hasta tal punto que aparecen nuevos perfiles profesionales vinculados con el diseño que antes no lo hubieran estado. Así, las técnicas de geolocalización o las de pragmática obtienen una relevancia en la confección de los mensajes cada vez mayor. 

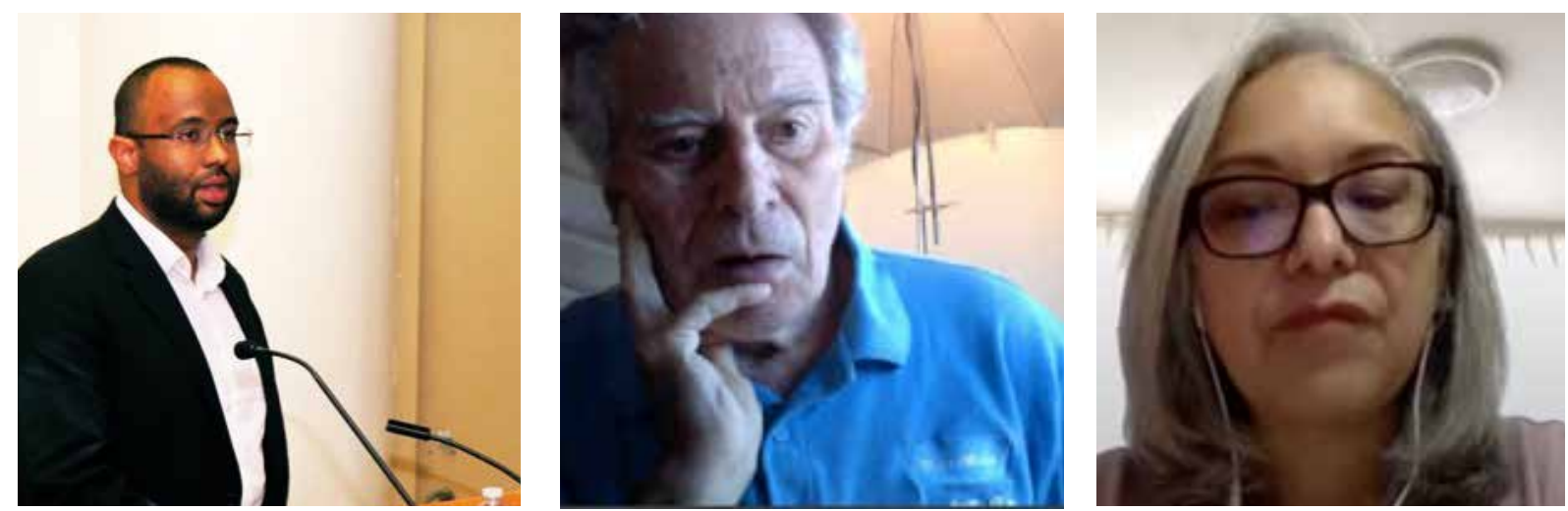

Figura 1. José Ayala, Figura 2. Javier González Solas; Figura 3. Teresa Camacho.

Análisis con Espíritu Crítico del Diseño. - Con este provocador título, Javier González Solas (figura. 2) se dispuso en su intervención a suscitarnos un sentido crítico ante la labor del diseño y de los diseñadores. Sus dotes de crítica reflejaron, como siempre hace, unas dosis de dudas existenciales hacia el trabajo que se hace desde el diseño, pero también y de una manera más descarnada, desde las instituciones. Prófugo de los límites ortodoxos del diseño y filósofo del trabajo del mismo, la conferencia versó sobre el rumbo que adoptó el diseño con la irrupción de los mecanismos del mercado y que sigue rigiendo los derroteros de los procedimientos seguidos por el diseño gráfico. Crítico con todo o casi todo, nos deleitó con su perspectiva de dar al diseño un sentido útil para ser comprendido como lo que es: una actividad al servicio de las personas.

Diseño, Diseñadores, Práctica i Docencia. - La vinculación de las palabras clave del título de la conferencia de Teresa Camacho (figura 3) nos retrotraen a la consideración del compromiso de todos los agentes participantes en la trayectoria del objeto diseñado con el compromiso con el propio diseño y con la sociedad. Ciertamente, las reflexiones de Teresa Camacho van en la orientación de consolidar, con precisión, el elemento central que debe regir el trabajo del diseño: el compromiso de diseño con la disciplina de estudio en las universidades y de si el diseño responde, responsablemente, a las necesidades sociales y contextuales. Eso introduce a las dimensiones del diseño en el sentido de su comprensión. Así podemos identificar las dimensiones del diseño: el proceso, el objeto y la persona. Y estas tres dimensiones deben conjugarse, además, en el terreno contextual en el que se insertan las propuestas del diseño.

\section{SUCEDIERON MÁS COSAS}

Y no se quedó ahí el VI Symposium grafica. Hubo 5 mesas dedicadas a investigación, 2 mesas dedicadas a docencia, y 1 mesa dedicada a los colegios profesionales en la que participaron Antonio Traugott por el colegio de Publicidad, Relaciones Públicas y Marketing de Catalunya y Jesús del Hoyo en representación de Rosa Fonts por el Colegio de Diseño Gráfico de Catalunya.

Además, se realizó la entrega, y consecuente ponencia de su autora, del II Premio grafica otorgado al artículo con más relevancia durante el periodo 2015-2019 y que ha sido publicado en grafica - International Journal of Graphic Design. Este año el Premio recayó en el artículo "Análisis de la comunicación visual del cartel cinematográfico. Estudio de caso de la productora Universal Pictures" de Irene Sánchez de Lucas. Corresponde a un paper publicado en el Volumen 6, No 12 (2018).

Presentamos a continuación la relación de ponencias y las autorías de las mismas: que fueron distribuidas según fuesen consideradas de Investigación, Didáctica o Herramientas:

\section{Investigación}

- Teoría sobre el Caos del Creativo · Rafael Pozo-Puértolas

- Los títulos de crédito: Su rol como packaging y huella visual de la película - Belén Ramírez-Barredo | Maria Luisa García-Guardia | Carmen Llorente-Barroso

- Las camisas con la marca de Salvador Dalí · Aida María de Vicente Domínguez 
- Modernización iconográfica religiosa dentro de un modelo de emprendimiento - Estíbaliz Vélez | Liceth Briceño | Edwin Chuico

- Diseño experimental en publicidad y diseño gráfico · David Badajoz

- Representación simbólica del sexo · Daniel Tena-Parera

- El poder del diseño para generar emociones · Sara Caldas

- La ley de Zipf en el lenguaje visual · Francesc Morera-Vidal

- La corbata, una buena forma · Mateu Batallé Canal | Patrícia Lázaro Pernias

— Diez puntos claves en la relación del diseño gráfico y la publicidad · Josep Rom

\section{Didáctica del diseño}

- Estrategias de aprendizaje online en situaciones especiales - José Manuel Martínez Bouza

- Adaptación de la docencia de la asignatura Color durante de crisis sanitaria del COVID . Bernardo Antonio Candela Sanjuán | Sergio Fernández Montañez-Madan

\section{Herramientas}

— La comunicación 2.0 de cosmética: Estudio del canal de YouTube de Dulceida · Pilar Vicente | Raquel Vinader

— Flash: 99\%... What? · Raquel Ávila Muñoz | Jorge Clemente Mediavilla | María José Pérez-Luque Maricalva

Los temas, ya dejan intuir que los debates surgidos fueron diversos y muy interesantes. Seguramente, el $7^{\circ}$ Symposiu grafica, que fue anunciado ya para el septiembre de 2021 y que tendrá la sede de I'Escola EINA, escuela adscrita a la Universitat Autònoma de Barcelona, recogerá todos los haberes del symposium y sabrá expandir más los éxitos de este encuentro.

\section{\# 27 Questiones Publicitarias}

El número 27 de Questiones Publicitarias (advertising questions) publica, dos artículos científicos, un ensayos y una reseña. Todas estas aportaciones orientadas respecto de nuestro objetivo de facilitar espacios de debate, de investigación y también de divulgación sobre las distintas cuestiones que el mundo de la publicidad, las relaciones públicas y la comunicación de las organizaciones.

El primer artículo de investigación lo firman los autores Marc Perez-Bonaventura, Salomé Tárrega y Jordi Vilajosana. Se trata de un trabajo conjunto de miembros de la colaboración entre dos Universidades: la Universitat Politècnica de Catalunya con el primer y tercer autor y la Universitat de Vic con el segundo. Los tres autores presentan el paper con el título "Las universidades de Madrid y Cataluña en redes socialesCatalan and Madrid universities in social media". La aportación presenta el contexto competitivo que tienen las instituciones universitarias en el marketing y la publicidad actuales. Son esenciales para las instituciones de educación superior. En el momento de la elección de universidad, los estudiantes hacen uso de las redes sociales. Por ello, el uso de esas redes es la mejor opción para que las instituciones de educación superior lleguen a los futuros alumnos. El presente artículo estudia el comportamiento en redes sociales de las universidades de Cataluña y la Comunidad de Madrid para tratar de atraer a los estudiantes y ser elegidas por ellos. Se pretende descubrir cuál de las dos comunidades tiene mejores resultados en las redes sociales.

El segundo de los artículos de investigación que publicamos corresponde a "La cultura participativa de Star Wars como experiencia transmedia". La aportación está firmada por Víctor Álvarez Rodríguez, de la Universidad de Cádiz, España. En este caso, se trata de un artículo sobre la figura de la cultura participativa en relación con una marca de ficción. El estudio busca conocer en detalle los procesos y decisiones tomadas por la organización de fans de Star Wars la Legión 501 para traer el mundo real parte de este relato desde una perspectiva física y de comportamiento. Este análisis desarrolla una metodología exploratorio-descriptiva dividida en fases. Como resultado, se identifican las dimensiones relacionadas con esta técnica, su identidad, y la experiencia transmedia generada gracias a la acción participativa.

Como complemento a estos dos "papers" presentamos un ensayo: "El papel de la técnica publicitaria Branded Content para generar nuevos vínculos de compromiso entre marca y 
sociedad". Su autora es María Rodríguez-Rabadán Benito, de la Universitat Internacional de la Rioja. El trabajo aborda la creación de contenidos de carácter informativo, educativo o de entretenimiento, al servicio de la marca presenta una oportunidad real en la generación de vínculos a medio y largo plazo con el consumidor y espectador. El artículo analiza las oportunidades que tienen las marcas para pasar a formar parte del cambio social de su tiempo comprometiéndose con las cuestiones importantes de su época. Una campaña publicitaria puede transformarse en una acción de impacto real en las vidas de los consumidores y es por ello que el Branded Content toma cada vez más relevancia.

Completa la entrega de esta edición la reseña "La imagen de las Relaciones Públicas a través de la Séptima Arte" realizada por Almudena Barrientos-Báez, de la Escuela Universitaria de Turismo Iriarte (ULL), España. El texto se hace eco del libro con el mismo nombre coordinado por Cabezuelo Lorenzo, F. y González Vallés, J. E.

Aunque las Relaciones públicas han sido analizadas desde variados puntos de vista: origen, funcionalidad, herramientas, utilidad... éste es el primer libro que las muestra no en su naturaleza interna sino a través del cine que no es sino esa parte de nuestra conciencia colectiva.

Solo nos queda agradecer a todos cuantos hacen posible este proyecto y esperar que este número sea del interés y utilidad para los lectores.

Buena y valiosa lectura.

REFERENCIAS BIBLIOGRÁFICAS

V.AA, Llibre col.lectiu 20symposium, Departament de Publicitat, Relacions Públiques i Comunicació Audiovisual (UAB), Barcelona, 2020

https://www.questionespublicitarias.es/

https://jornades.uab.cat/symposiumgrafica/es 
